\title{
Case Report of a Pelvic Mass; Bladder Diverticulum
}

\section{A R T I C L E I N F O}

\section{Article Type}

Case Report

\section{Authors}

Saremi A.B. ${ }^{1} M D$

Rasekhi M.*MD

\section{How to cite this article} Saremi A B, Rasekhi M. Case Report of a Pelvic Mass; Bladder Diverticulum. Sarem Journal of Reproductive Medicine. 2017;1(4): 191-194.

\begin{abstract}
A B S T R A C T
Patient Information One of the diseases of the urinary system is bladder diverticulum. Bladder diverticula are seen in different sizes, individual or multiple. These lesions may be congenital and create symptoms during childhood, which are usually diagnosed during this period, or may be acquired, resulting from narrowing of the urinary tract from the bladder (for example, prostate hypertrophy). Occasionally, diverticula are diagnosed in abdominal and pelvic imaging procedures, without causing a problem for the patient.

The case reported is a bloody diverticulum presented as pelvic mass and not detected before surgery. The patient suffered from abdominal pain and did not describe the symptoms of urinary tract infection. In the imaging procedures, the pelvic mass has been reported without communication with the bladder, and only during laparoscopy, we noticed the association of mass with the bladder, and the patient was subjected to diverticulectomy.

Conclusion Diseases do not necessarily reveal themselves with the typical symptoms mentioned in the texts, and the important point is that paraclinical methods are not always accurate and recognizable. Laparoscopy can be considered as a precise diagnostic method and fully reveals the organs of the abdomen and pelvis.
\end{abstract}

Keywords Diverticulum; Pelvic; Laparoscopy; Diagnostic Imaging
*Sarem Fertility and Infertility Research Center (SAFIR), Sarem Women's Hospital, Tehran, Iran ${ }^{1 " S a r e m ~ F e r t i l i t y ~ \& ~ I n f e r t i l i t y ~ R e-~}$ search Center (SAFIR)" and "Sarem Cell Research Center (SCRC)", Sarem Women's Hospital, Tehran, Iran

\section{Correspondence}

Address: Sarem Women's Hospital, Basij Square, Phase 3, Ekbatan Town, Tehran, Iran. Postal Code: 1396956111

Phone: +98 (21) 44670888

Fax: +98 (21) 44670432

m.rasekhi10@gmail.com

\section{Article History}

Received: April 24, 2016

Accepted: October 16, 2016

ePublished: November 15, 2017

\section{I T A T I O N L I N K S}

[1] Vaginal mass caused by a lately recognized congenital bladder diverticulum [2] The aetiology and treatment of diverticulum of the bladder [3] Syncope in a patient with giant bladder diverticulum [4] Extravesical approach in paraureteral bladder diverticulum: A case report[5] Management of congenital bladder diverticulum in children: A report of seven cases [6] Congenital bladder diverticulum causing acute urinary retention in an infant [7] Congenital bladder diverticulum- a rare adult presentation [8] Congenital genitourinary abnormalities in children with Williams-Beuren syndrome [9] Urethral obstruction from dislodged bladder diverticulum stones: A case report [10] Ruptured bicornuate uterus mimicking ectopic pregnancy: A case report [11] An atypical bladder diverticulum presented with recurrent peritonitis: Case report [12] Female urethral diverticulum presenting with acute urinary retention: Reporting the largest diverticulum with review of literature [13] Bladder diverticulum: Clinicopathologic spectrum in pediatric patients [14] Case of congenital bladder diverticulum--cause of urinary retention in adulthood [15] Median umbilical ligament or vesicourachal diverticulum [16] Intravesical methylene blue facilitates precise identification of the diverticular neck during robot-assisted laparoscopic bladder diverticulectomy 
وجود دارد يا نه مشخص نيست، ولى جون اين عارضه دار دور دوقلوها گزارش شده احتمالاً جنبه اتوزوم غالب داردة داردة].

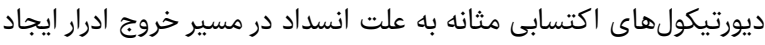

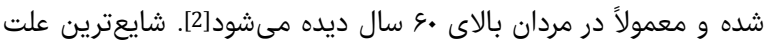

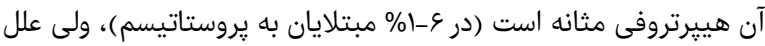

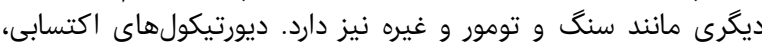

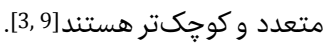

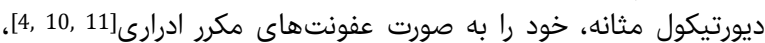
احساس عدم تخليه كامل ادرار[2]

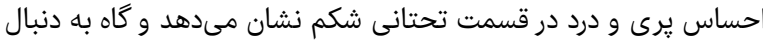

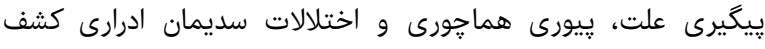

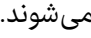
براى تشخيص اين بيمارى سونوگرافى، سىتىاسكن و امآرآى درخواست

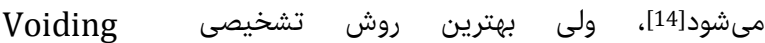
Cystouetrography (VCUG)

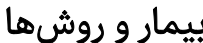

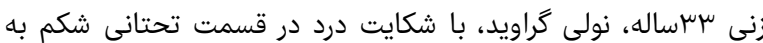

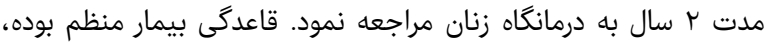

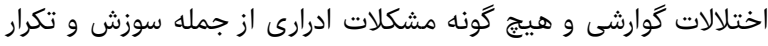

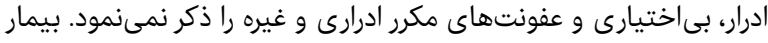

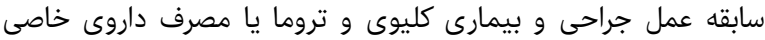

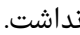

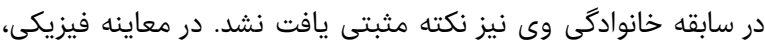

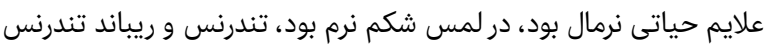

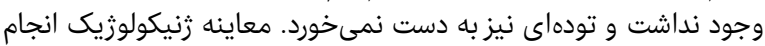

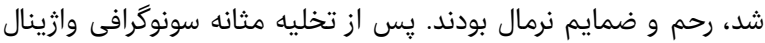

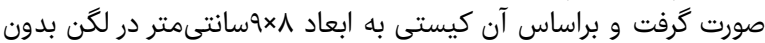

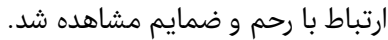

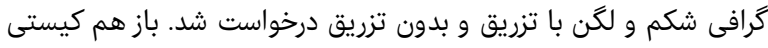

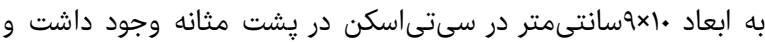

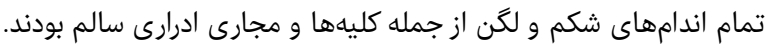

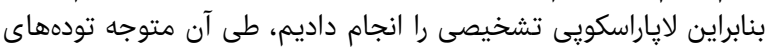

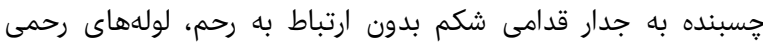

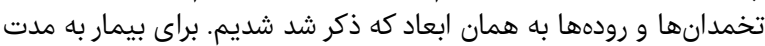

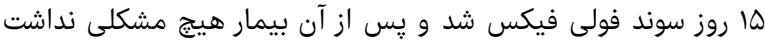

به نظر مىرسيد كه توده در مسير باشد[15]، ولى با بررسى دقيقتر متوجه

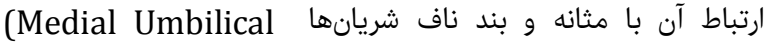

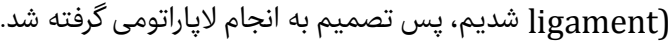

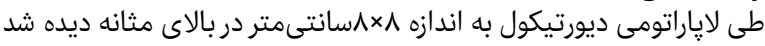

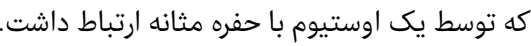

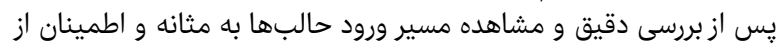

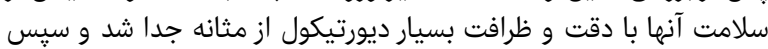

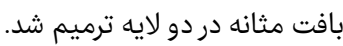

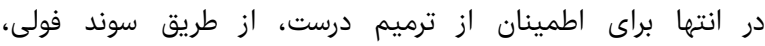

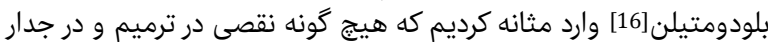
دوختهشده وجود نداشت.

كزارش ذكرشده مورد نادرى از ديورتيكول مثانه و نيز مورد نادرى در تشخيص افتراقى تودههاى لخنى است.

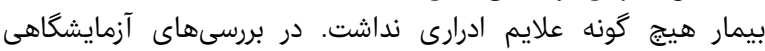

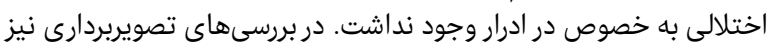
سيستم ادرارى سالم گزارش شده بورار بود ندا
كزارش يك مورد توده لخنى با تشخيص نهايى ديورتيكول مثانه

\author{
MD ابوطالب صارمى تحقيق باروى
}

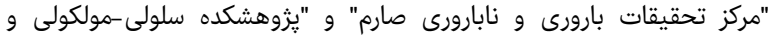

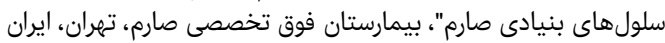

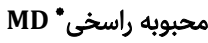
مركز تحقيقات بارورى و نابارورى صارم، بيمارستان فوق تخصصى صارم، تهران،

جكيده مقدمه: يكى از بيدمارىهاى سيستم ادرارى، ديورتيكولهاى مثانه ماسيت.

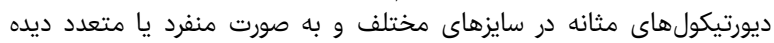

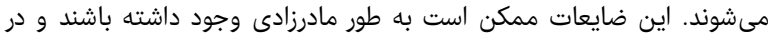

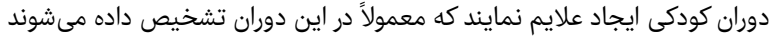

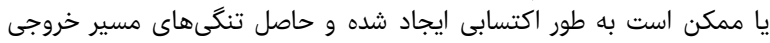

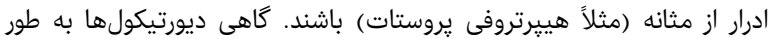

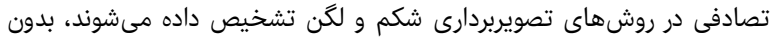

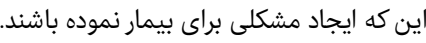

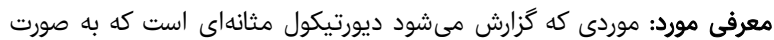

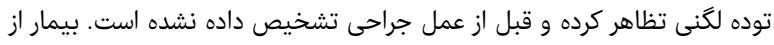

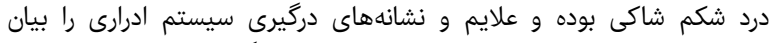

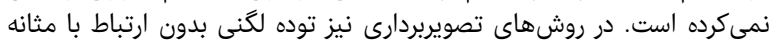

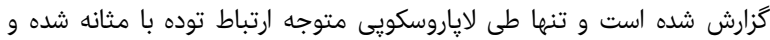

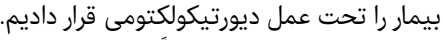

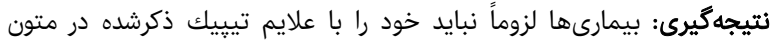

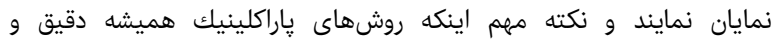

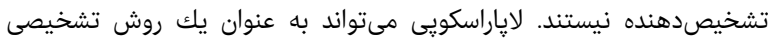

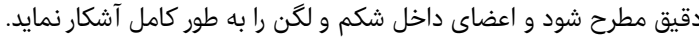

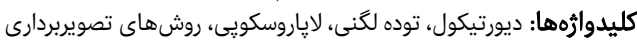

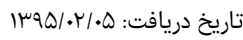

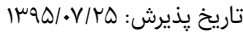

m.rasekhi10@gmail.com :تويسنده مسئول:

مقدمه

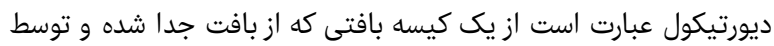

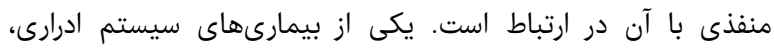

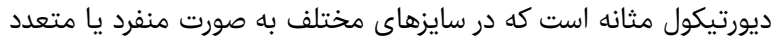

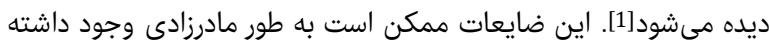

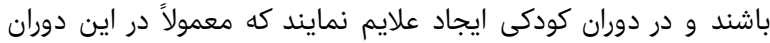

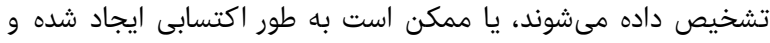

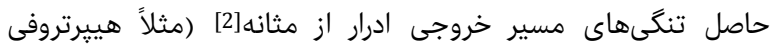

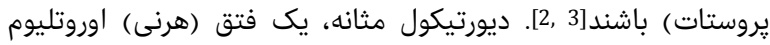

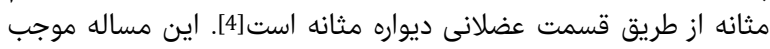

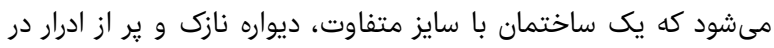

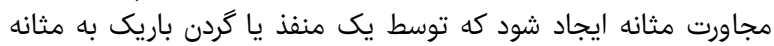

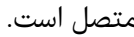

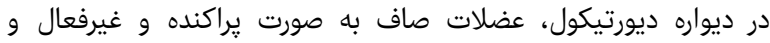

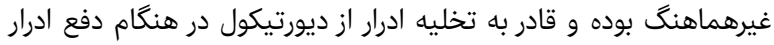

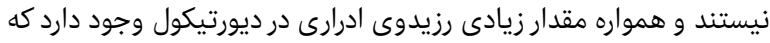

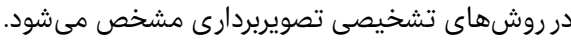

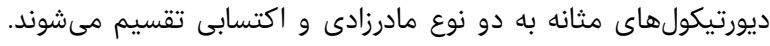

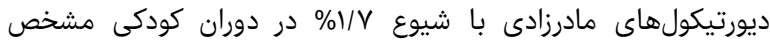

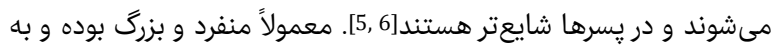

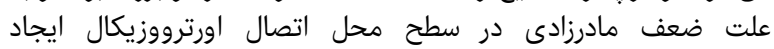
مىشوند[1, 11. اينكه در نوع مادرزادى بادي شيوع ديورتيكول اختلال زنتيك ايجال 
نتيجه گيرى

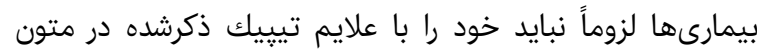

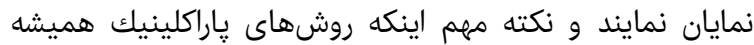

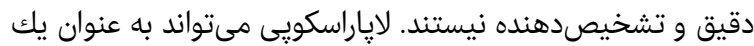

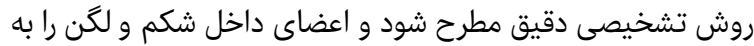

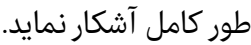

تشكر و قدردانى: از همكاران محترم اتاق عمل بيمارستان فوق تخصصى صارم تشكر مى شود.

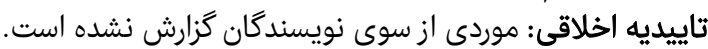
تعارض منافع: موردى وجود نداشتيه اخون است.

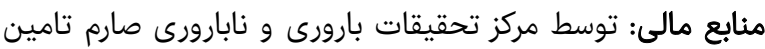

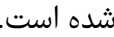
سهم نويسندگان: ابوطالب صارمى (نويسنده اول)، نكارنده

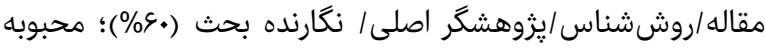

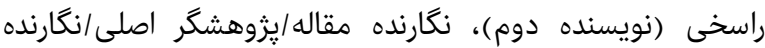

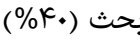

منابع

1- Zengin K, Tanik S, Gürdal M, Okur A, Zengin B. Vaginal mass caused by a lately recognized congenital bladder diverticulum. Can Urol Assoc J. 2013;7(9-10):E634-6.

2- Miller A. The aetiology and treatment of diverticulum of the bladder. Br J Urol. 1958;30(1):43-56.

3- Kaneko N, Kawasaki T, Boku H, Kamitani T. Syncope in a patient with giant bladder diverticulum. Intern Med. 2012;51(14):1935-6.

4- Moralioglu S, Bosnali O, Celayir AC, Sahin C. Extravesical approach in paraureteral bladder diverticulum: A case report. West Indian Med J. 2014;63(2):201-3.

5- Khemakhem R, Ghorbel S, Jlidi S, Nouira F, Louati H, Douira $\mathrm{W}$, et al. Management of congenital bladder diverticulum in children: A report of seven cases. Afr J Paediatr Surg. 2013;10(2):160-3.

6- Hossain MZ, Hasan GZ, Siddiqui TH. Congenital bladder diverticulum causing acute urinary retention in an infant. Mymensingh Med J. 2012;21(2):360-2.

7- Vaddi S, Pogula VR, Devraj R, Sreedhar AV. Congenital bladder diverticulum- a rare adult presentation. J Surg Case Rep. 2011;2011(4):8.

8- Sammour ZM, Gomes CM, de Bessa J Jr, Pinheiro MS, Kim CA, Hisano $M$, et al. Congenital genitourinary abnormalities in children with Williams-Beuren syndrome. J pediatr urol. 2014;10(5):804-9.

9- Okeke LI, Takure AO, Adebayo SA, Oluyemi OY, Oyelekan AAA. Urethral obstruction from dislodged bladder diverticulum stones: A case report. BMC Urol. 2012;12:31.

10- Singh N, Singh U, Verma ML. Ruptured bicornuate uterus mimicking ectopic pregnancy: A case report. The J Obstet Gynaecol Res. 2013;39(1):364-6.

11- Temiz A, Akçora B, Atik E. An atypical bladder diverticulum presented with recurrent peritonitis: Case report. Ulus Travma Acil Cerrahi Derg. 2011;17(4):3657.

12- Pradhan MR, Ranjan P, Kapoor R. Female urethral diverticulum presenting with acute urinary retention: Reporting the largest diverticulum with review of

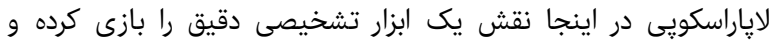

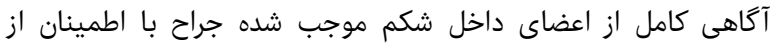

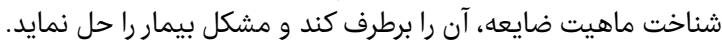

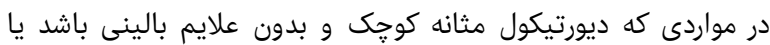

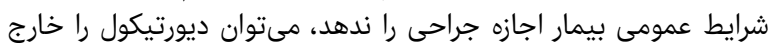

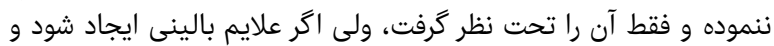

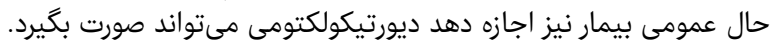

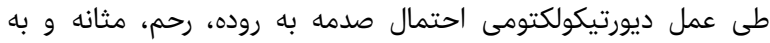

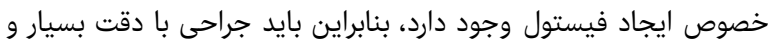
ترجيحاً لاياراتومى صورت گيرد.
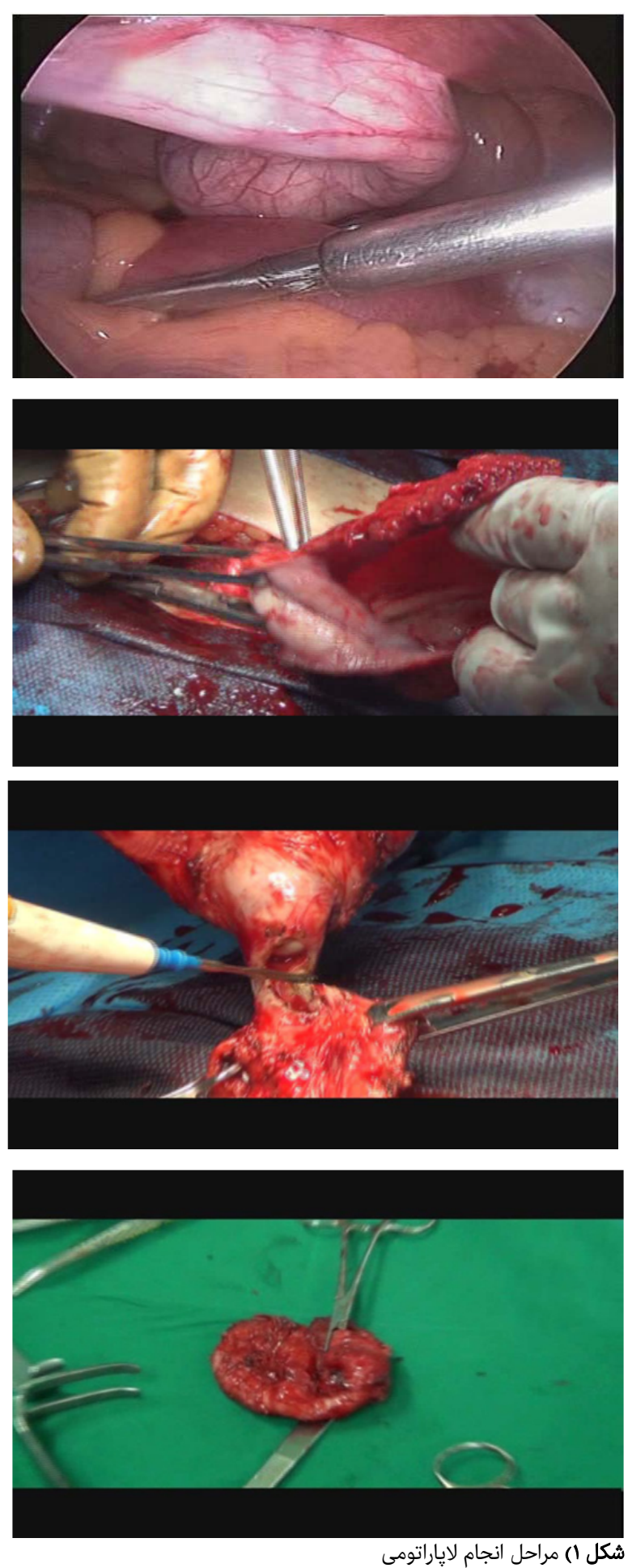
15- Cho FN, Liu CB. Median umbilical ligament or vesicourachal diverticulum. J Minim Invasive Gynecol. 2012;19(2):150-1.

16- Moore CR, Shirodkar SP, Avallone MA, Castle SM, Gorin MA, Gorbatiy V, et al. Intravesical methylene blue facilitates precise identification of the diverticular neck during robot-assisted laparoscopic bladder diverticulectomy. J Laparoendosc Adv Surg Tech A. 2012;22(5):492-5. literature. Indian J Urol. 2012;28(2):216-8.

13- Alexander RE, Kum JB, Idrees M. Bladder diverticulum: Clinicopathologic spectrum in pediatric patients. Pediatr Dev Pathol. 2012;15(4):281-5.

14- Fujita N, Tambo M, Shishido T, Okegawa T, Nutahara $\mathrm{K}$, Higashihara $\mathrm{E}$. Case of congenital bladder diverticulum--cause of urinary retention in adulthood. Nihon Hinyokika Gakkai Zasshi. 2010;101(4):619-21. [Japanese] 